\title{
Evolutionary role of chemotherapy in advanced
} nasopharyngeal carcinoma: a literature-based network meta-analysis

This article was published in the following Dove Medical Press journal: Cancer Management and Research

\author{
$\mathrm{Li} \mathrm{Li}$ \\ Wei Liang' \\ Jin-Xian Zhu' \\ Chun-Jie Dong ${ }^{2}$ \\ Yuan-Mei Zou' \\ Bi-Cui Ye' \\ Lin Gao'
}

'Department of Radiation Oncology, Wuzhou Red Cross Hospital, Wuzhou, Guangxi, People's Republic of China; ${ }^{2}$ Department of Internal Medicine, The Second Hospital of Fushun, Fushun, Liaoning, People's Republic of China
Correspondence: $\mathrm{Li} \mathrm{Li}$

Department of Radiation Oncology, Wuzhou Red Cross Hospital, 3-I Xinxing First Road, Wuzhou 54300I, Guangxi,

People's Republic of China

Tel +8607743832052

Email leeli8135@sina.com
Purpose: The role of chemotherapy has evolved greatly in advanced nasopharyngeal carcinoma (NPC). We undertook this network meta-analysis to establish the optimal chemotherapy strategy in advanced NPC.

Materials and methods: This network meta-analysis recruited randomized clinical trials involving patients with advanced NPC randomly allocated to induction chemotherapy plus concurrent chemoradiotherapy (CRT; induction + CRT), CRT plus adjuvant chemotherapy (CRT + adjuvant), CRT or radiotherapy (RT) alone. Pairwise meta-analysis was first conducted, then network meta-analysis was performed using the frequentist approach. Effect size was expressed as $\mathrm{HR}$ and $95 \% \mathrm{CI}$.

Results: In total, we analyzed 15 studies involving 4,067 patients with 880 (21.6\%) patients receiving induction + CRT, 897 (22.1\%) receiving CRT + adjuvant, 1,421 (34.9\%) receiving CRT, and 869 (21.4\%) receiving RT alone. Induction + CRT achieved significantly better distant failure-free survival (HR, 0.67; 95\% CI, 0.53-0.86) and locoregional failure-free survival (HR, $0.69 ; 95 \%$ CI, 0.54-0.89) than CRT, and CRT + adjuvant achieved better overall survival than CRT (HR, 0.82; 95\% CI, 0.67-1.00). However, no significant survival difference was found between the induction + CRT and CRT + adjuvant groups. Additionally, RT alone is always worse than the other three treatments. In terms of P-score, induction + CRT ranked best for distant and locoregional failure-free survival, while CRT + adjuvant ranked best for overall survival. Conclusion: Both induction + CRT and CRT + adjuvant were equally effective and feasible choices for patients with advanced NPC.

Keywords: nasopharyngeal carcinoma, advanced, concurrent chemoradiotherapy, induction chemotherapy, adjuvant chemotherapy, network meta-analysis

\section{Introduction}

Nasopharyngeal carcinoma (NPC), known as a special kind of head and neck malignancy, is mainly prevalent in East Asia and South China while its incidence in white population is extremely low. ${ }^{1}$ According to the recent data on cancer epidemiology, NPC has emerged as the most common head and neck cancer in China. ${ }^{2,3}$ Radiotherapy (RT) is the only radical therapy for nonmetastatic disease as a result of complicated anatomy location and high radiation sensitivity of NPC. Also, NPC is highly sensitive to chemotherapy and combined RT with chemotherapy is essential for advanced disease.

The role of chemotherapy in advanced NPC was first established by the Intergroup 0099 study, which revealed concurrent prevalent (CRT) plus adjuvant chemotherapy achieved better overall survival (OS) than RT alone. ${ }^{4}$ Later on, many validated trials 
that were carried out in Asia further strengthen the role of CRT plus adjuvant chemotherapy in NPC. ${ }^{5-7}$ However, a randomized phase III clinical trial by Chen et al demonstrated that adjuvant chemotherapy additional to CRT may be useless. ${ }^{8}$ Consequently, CRT with or without adjuvant chemotherapy has been recommended as the standard treatment for advanced NPC. Although these achievements have been made, the prognosis of advanced disease still remains poor. ${ }^{9}$ Therefore, scientists evaluated the efficacy and toxicity of induction chemotherapy additional to CRT in advanced NPC. Thankfully, recent studies found that induction chemotherapy plus CRT was superior to CRT alone, ${ }^{10-14}$ making induction chemotherapy a promising treatment for advanced NPC. However, another clinical issue produced: what is the best treatment in advanced NPC? Induction chemotherapy plus CRT (induction + CRT) or CRT plus adjuvant chemotherapy (CRT + adjuvant)? To date, no head-to-head clinical study comparing induction + CRT with CRT + adjuvant has been reported. A network meta-analysis employing individualized patient data (IPD) compared these two treatments indirectly and found no significant difference. ${ }^{15}$ Notably, many recent studies were not included in this meta-analysis. Thus, it is worth reanalyzing this issue using the updated data. Given this concern, we conducted this network meta-analysis to establish the optimal treatment in advanced NPC.

\section{Materials and methods \\ Online literature searching strategy}

We searched the online datasets of PubMed, Web of Science, and Cochrane library using the terms of "nasopharyngeal carcinoma or cancer," "radiotherapy," and "induction chemotherapy or neoadjuvant chemotherapy or adjuvant chemotherapy or concurrent chemoradiotherapy" to identify all potential clinical trials. For related Chinese studies, the National Knowledge Infrastructure and WanFang datasets were searched. All studies were restricted to randomized clinical trial. Two investigators completed this process independently, and any discrepancy was solved by consensus. Our network meta-analysis was conducted in accordance with the PRISMA guidelines (PRISMA checklist).

\section{Clinical trial inclusion criteria}

The clinical trials published between 1998 and 2018 would be included into this study for analysis if they meet the following criteria: 1) randomized phase II-III clinical trial; 2) recruiting patients with newly diagnosed, nonmetastatic, and advanced NPC; 3) RT was delivered as conventional fraction and total radiation dosage of 66 Gy or more should be scheduled; 4) either experimental or control arm in the trials should contain one of the four treatments (induction + CRT, CRT + adjuvant, CRT alone, RT alone).

\section{Study quality evaluation}

In order to select appropriate statistical method and obtain unbiased results, we employed the Jadad/Oxford quality scoring system ${ }^{16}$ to assess the study quality. The randomization procedure, sample size calculation, adoption of blinded principle, allocation concealment, and intention-to-treatment analysis of included trials were reviewed and scored according to the standard. Two authors completed this process independently and discrepancies were solved by consensus.

\section{Study data extraction}

Three investigators reviewed each included study separately to collect and extract related data including study author information, patient recruitment time period, sample size, patient tumor stage, RT and chemotherapy protocol, followup duration, and study endpoints. All extracted information and data were reviewed by the fourth investigator to check whether discrepancy existed between the three investigators. Otherwise, discrepancy would be solved by consensus.

\section{Study endpoint}

In our current network meta-analysis, we set OS (defined as the time interval between randomization and death from any cause) as the primary endpoint. The other two endpoints were distant failure-free survival (DFFS, defined as time interval between randomization to first distant metastasis) and locoregional failure-free survival (LFFS, defined as time interval between randomization and first local or regional or both recurrence). Given the different definitions of progression-free survival (PFS) or disease-free survival in different clinical trials, we therefore did not perform analysis on this endpoint.

\section{Statistical analysis}

Survival data were extracted from trials and expressed in our study as HRs and corresponding CIs since they are the only summary statistic allowed for censoring and time to an event. HRs and corresponding CIs were extracted from original text if they were available, otherwise they were obtained them from a previous meta-analysis ${ }^{15}$ or a pooled data analysis. ${ }^{17}$

Pairwise meta-analysis between two treatment arms was conducted first. Pooled HRs and corresponding 95\% CIs of direct comparison between two treatment arms were calculated to evaluate the survival difference, and $P<0.05$ was 
considered significant. We used the chi-squared test and $I^{2}$ statistic to establish the heterogeneity between studies, and the $\chi^{2} P$-value $<0.1$ or an $I^{2}$ statistic $>50 \%$ was considered significant. Stata statistical package 13.0 (StataCorp LP, College Station, TX, USA) was applied to complete this analysis.

For network meta-analysis, multiple treatment comparisons were conducted using netmeta package ${ }^{18,19}$ and frequentist approach ${ }^{18}$ in R software (version 3.3.5; R Foundation, Vienna, Austria). The logarithmic of HR (logHR) and its variance (selogHR) of each direct comparison were calculated for statistical network meta-analysis. Also, treatment effects of network meta-analysis were estimated by HRs and 95\% CIs and presented in forest plots. Inconsistency and heterogeneity between and within different comparisons were evaluated by $Q$ test, which was proposed by Rücker. ${ }^{19}$ The $P$-value of $Q$ test $>0.1$ indicates no heterogeneity, and vice versa. Random-effect model would be applied and sensitivity analysis would be performed in case of significant heterogeneity. Finally, each treatment would be ranked by a P-score, which was proposed by Rücker and Schwarzer ${ }^{18}$ as a frequentist analog to surface under the cumulative ranking curve. $^{20,21}$ Briefly, a P-score of $100 \%$ indicates the best treatment and $0 \%$ for the worst treatment. $P<0.05$ was considered significant for all analysis.

\section{Results}

\section{Basic information of included studies}

By the last searching (August 2018), we totally identified 26 potentially eligible clinical trials and the flowchart is presented in Figure S1. The study by Lin et $\mathrm{al}^{22}$ comparing CRT with RT alone was excluded because HRs and 95\% CIs were not provided in the original text. We also excluded two studies which recruited patients with stage II disease..$^{23,24}$ Moreover, the study by Lee et $\mathrm{al}^{25}$ consisting of six treatment arms was not included because HR was not provided for each comparison. Two treatment arms in the study by Lee et $\mathrm{al}^{26,27}$ receiving accelerated-fraction RT were not included but the other arms were included. Nevertheless, six studies updated their follow-up data: Chen et al comparing CRT + adjuvant with CRT, ${ }^{28,29}$ Chan et al comparing CRT with RT, ${ }^{30,31}$ Lee et al comparing CRT + adjuvant with $\mathrm{RT},{ }^{6,32}$ Chen et al comparing CRT + adjuvant with RT, ${ }^{33,34}$ Lee et al comparing CRT + adjuvant with RT, ${ }^{26,27}$ and Zhang et al and Wu et al comparing CRT with RT. ${ }^{35,36}$ Finally, 15 studies were included for our study ${ }^{4-8,10-14,35,37-40}$ and the basic information is shown in Table 1. Overall, these 15 trials recruited 4,067 patients with $880(21.6 \%)$ patients receiving induction + CRT, 897 (22.1\%) receiving CRT + adjuvant, 1,421 (34.9\%) receiving CRT, and
869 (21.4\%) receiving RT alone. The Jadad/Oxford score for each trial is summarized in Table S1. Obviously, most of the trials achieved good quality.

\section{Pairwise meta-analysis between two treatment arms}

Figure 1 presents the results of all direct comparisons. As shown by the result, no significant heterogeneity exists between all comparisons for all the endpoints. Therefore, the fixed-effect model was employed for all analysis. Compared with RT alone, CRT + adjuvant achieved significantly better OS (HR, 0.63; 95\% CI, 0.53-0.73), DFFS (HR, 0.51; 95\% CI, 0.39-0.64), and LFFS (HR, 0.48; 95\% CI, 0.32-0.64), while CRT was associated with better OS (HR, 0.77; 95\% CI, 0.61-0.93) and DFFS (HR, 0.56; 95\% CI, 0.39-0.74) but not LFFS (HR, 0.81; 95\% CI, 0.50-1.11). As expected, no significant difference was found between CRT + adjuvant and CRT. Moreover, induction + CRT achieved significantly better OS (HR, 0.74; 95\% CI, 0.57-0.91), DFFS (HR, 0.65; 95\% CI, 0.50-0.80), and LFFS (HR, 0.66; 95\% CI, 0.48-0.84).

\section{Network comparison between multiple treatment arms}

The network plot of multiple treatment comparisons is presented in Figure 2. No significant between-study or withinstudy heterogeneity was found between all comparisons (Table 2), and the fixed-effect model was therefore applied. The forest plot of multiple treatment comparisons in the network meta-analysis is shown in Figure 3.

Compared with CRT, CRT + adjuvant achieved significantly better OS (HR, 0.82; 95\% CI, 0.67-1.00), while RT alone achieved significantly worse OS (HR, 1.25; 95\% CI, 1.05-1.49). However, no statistical difference was found between induction + CRT and CRT (HR, 0.83; 95\% CI, 0.67-1.03). After changing the reference group, CRT + adjuvant did not significantly differ from induction + CRT (HR, 1.02; 95\% CI, 0.76-1.36); while RT alone was poorer than both CRT + adjuvant (HR, 1.53; 95\% CI, 1.32-1.77) and induction + CRT (HR, 1.50; 95\% CI, 1.14-1.98). The P-scores for CRT, CRT + adjuvant, induction + CRT, and RT alone were $35.3 \%, 84.1 \%, 80.3 \%$, and $0.3 \%$, respectively (Table 2), suggesting that CRT + adjuvant achieved the higher possibility of becoming the best treatment for OS.

For the endpoint of DFFS, induction + CRT was better than CRT (HR, 0.67; 95\% CI, 0.53-0.86), while no significant difference was found between CRT and CRT + adjuvant (HR, 0.94; 95\% CI, 0.70-1.28) or between CRT + adjuvant and induction + CRT (HR, 0.71; 95\% CI, 0.48-1.05). Notably, RT 
Table I Basic information of the 15 clinical trials included

\begin{tabular}{|c|c|c|c|c|c|}
\hline Author & $\begin{array}{l}\text { Recruitment } \\
\text { time }\end{array}$ & $\begin{array}{l}\text { Sample size } \\
\text { (experimental/ } \\
\text { control) }\end{array}$ & $\begin{array}{l}\text { Tumor } \\
\text { stage }\end{array}$ & $\begin{array}{l}\text { Median } \\
\text { follow-up }\end{array}$ & Radiotherapy protocol \\
\hline \multicolumn{6}{|l|}{$\begin{array}{l}\text { CRT + adjuvant } \\
\text { vs RT }\end{array}$} \\
\hline Al-Sarraf et $\mathrm{al}^{4}$ & $1989-1995$ & $78 / 69^{a}$ & III-IV & $32.4 \mathrm{~m}$ & $66-70$ Gy at I.8-2.0 Gy/f/day (5 f/qw) \\
\hline Kwong et $\mathrm{al}^{38 \mathrm{~b}}$ & $|995-200|$ & $57 / 55$ & II-IV & $37 \mathrm{~m}$ & $\begin{array}{l}66-68 \text { Gy at } 2.0 \text { or } 2.5 \mathrm{~Gy} / \mathrm{f} / \text { daily ( } 4 \text { or } 5 \mathrm{f} / \mathrm{qw})+ \\
10 \mathrm{~Gy} \text { boost dose to parapharyngeal extension or } \\
\text { residual neck node }\end{array}$ \\
\hline Wee et $\mathrm{al}^{7}$ & $1997-2003$ & $111 / 110$ & $\begin{array}{l}\text { III-IV, T3-4Nx or } \\
\text { TxN2-3 }\end{array}$ & $38.4 \mathrm{~m}$ & $70 \mathrm{~Gy} / 35 \mathrm{f}$ at $2 \mathrm{~Gy} / \mathrm{f} /$ day $(5 \mathrm{f} / \mathrm{qw}$ ) for 7 weeks \\
\hline Lee et $\mathrm{al}^{6}$ & 1999-2004 & $172 / 178$ & III-IV, N2-3 & $70.8 \mathrm{~m}$ & $\begin{array}{l}\geq 66 \mathrm{~Gy} \text { at } 2.0 \mathrm{~Gy} / \mathrm{f} / \text { day }(5 \mathrm{f} / \mathrm{qw})+\text { additional boosts } \\
\text { to parapharyngeal space, primary or nodal sites } \\
\text { when indicated not exceeding } 20 \mathrm{~Gy}\end{array}$ \\
\hline Lee et $\mathrm{a}^{26}$ & 1999-2004 & $51 / 42$ & III-IV, T3-4N0-I & $75.6 \mathrm{~m}$ & $\begin{array}{l}\geq 66 \mathrm{~Gy} \text { at } 2.0 \mathrm{~Gy} / \mathrm{f} / \text { day }(5 \mathrm{f} / \mathrm{qw})+\text { additional boosts } \\
\text { to parapharyngeal space, primary or nodal sites } \\
\text { when indicated not exceeding } 20 \mathrm{~Gy}\end{array}$ \\
\hline Chen et $\mathrm{al}^{5}$ & $2002-2005$ & $158 / 158$ & $\begin{array}{l}\text { III-IV, TI-4 or } \\
\text { N0-3 }\end{array}$ & $70 \mathrm{~m}$ & $\begin{array}{l}\geq 68 \mathrm{~Gy} \text { at } 2.0 \mathrm{~Gy} / \mathrm{f} / \mathrm{day}(5 \mathrm{f} / \mathrm{qw}) \text { for } 7 \text { weeks } \\
+ \text { additional boost in case of parapharyngeal } \\
\text { extension, residual neck and/or nasopharyngeal } \\
\text { tumor }\end{array}$ \\
\hline \multicolumn{6}{|l|}{ CRT vs RT } \\
\hline Chan et $\mathrm{al}^{30}$ & $1994-1997$ & $174 / 176$ & $\begin{array}{l}\text { Ho's } \mathrm{N} 2-3 \text { or } \mathrm{NI} \\
\text { with nodal size } \\
\geq 4 \mathrm{~cm}\end{array}$ & $66 \mathrm{~m}$ & $\begin{array}{l}66 \mathrm{~Gy}+\text { additional boost in case of parapharyngeal } \\
\text { extension, residual neck or nasopharyngeal tumor }\end{array}$ \\
\hline Kwong et $\mathrm{al}^{38 \mathrm{~b}}$ & $|995-200|$ & $56 / 55$ & II-IV & $37 \mathrm{~m}$ & $\begin{array}{l}66-68 \text { Gy at } 2.0 \text { or } 2.5 \mathrm{~Gy} / \mathrm{f} / \text { daily ( } 4 \text { or } 5 \mathrm{f} / \mathrm{qw})+ \\
10 \mathrm{~Gy} \text { boost dose to parapharyngeal extension or } \\
\text { residual neck node }\end{array}$ \\
\hline Zhang et $\mathrm{a}^{36}$ & $200 \mathrm{I}-2003$ & $59 / 56$ & III-IV, N2-3 & $114 \mathrm{~m}$ & $\begin{array}{l}70-74 \mathrm{~Gy} \text { at } 2 \mathrm{~Gy} / \mathrm{f} / \mathrm{day}(5 \mathrm{f} / \mathrm{qw})+\text { additional boost } \\
\text { in case of parapharyngeal extension, residual neck } \\
\text { or nasopharyngeal tumor }\end{array}$ \\
\hline \multicolumn{6}{|l|}{$\begin{array}{l}\text { Induction + CRT } \\
\text { vs CRT }\end{array}$} \\
\hline Hui et $\mathrm{al}^{13}$ & $2002-2004$ & $34 / 31$ & III-IVB, TI-4, N0-3 & $51.6 \mathrm{~m}$ & $\begin{array}{l}66 \mathrm{~Gy} / 33 \mathrm{f} \text { at } 2 \mathrm{~Gy} / \mathrm{f} / \mathrm{day}(5 \mathrm{f} / \mathrm{qw})+\text { additional boost } \\
\text { of } 20 \mathrm{~Gy} / 10 \mathrm{f} \text { to parapharyngeal }\end{array}$ \\
\hline Tan et $\mathrm{al}^{40}$ & 2004-2012 & $86 / 86$ & III-IVB, TI-4, N0-3 & $40.8 \mathrm{~m}$ & $\begin{array}{l}\text { 2D-RT: } 70 \mathrm{~Gy} / 35 \mathrm{f} \text { at } 2 \mathrm{~Gy} / \mathrm{f} / \mathrm{day}(5 \mathrm{f} / \mathrm{qw}) \\
\text { IMRT: } 69.96 \mathrm{~Gy} / 33 \mathrm{f} \text { at } 2.12 \mathrm{~Gy} / \mathrm{f} / \text { day ( } 5 \mathrm{f} / \mathrm{qw})\end{array}$ \\
\hline Sun et $\mathrm{al}^{\prime \prime}$ & $2011-2013$ & $241 / 239$ & $\begin{array}{l}\text { III-IVB, except } \\
\text { T3-4N0 }\end{array}$ & $45 \mathrm{~m}$ & $\geq 66$ Gy at $2.00-2.35$ Gy/f/day for $6-7$ weeks \\
\hline Cao et $\mathrm{al}^{10}$ & $2008-2015$ & 476 & $\begin{array}{l}\text { III-IVB, except } \\
\text { T3N0-I }\end{array}$ & $50 \mathrm{~m}$ & $\geq 66$ Gy at $2.0-2.33 \mathrm{~Gy} / \mathrm{f} / \mathrm{day}$ \\
\hline Frikha et al ${ }^{12}$ & $2009-2012$ & $42 / 41$ & T2b-4 and/or NI-3 & $43.1 \mathrm{~m}$ & 70 Gy at 2 Gy/f/day (5 f/qw) \\
\hline Hong et $\mathrm{al}^{14}$ & 2003-2009 & $239 / 240$ & IVA-IVB & $72.0 \mathrm{~m}$ & $\begin{array}{l}\text { At least } 70 \text { Gy at I.8-2.0 Gy/f/day ( } 5 \text { f/qw) for } \\
33-39 \mathrm{f}\end{array}$ \\
\hline \multicolumn{6}{|l|}{$\begin{array}{l}\text { CRT + adjuvant } \\
\text { vs CRT }\end{array}$} \\
\hline Kwong et $\mathrm{al}^{38 \mathrm{~b}}$ & $|995-200|$ & $57 / 56$ & II-IV & $37 \mathrm{~m}$ & $\begin{array}{l}66-68 \mathrm{~Gy} \text { at } 2.0 \text { or } 2.5 \mathrm{~Gy} / \mathrm{f} / \mathrm{daily}(4 \text { or } 5 \mathrm{f} / \mathrm{qw})+ \\
10 \mathrm{~Gy} \text { boost dose to parapharyngeal extension or } \\
\text { residual neck node }\end{array}$ \\
\hline Chen et $\mathrm{al}^{8}$ & $2006-2010$ & $251 / 257$ & $\begin{array}{l}\text { III-IVB except } \\
\text { T3-4N0 }\end{array}$ & $68.4 \mathrm{~m}$ & $\geq 66$ Gy at $2.0-2.27 \mathrm{~Gy} / \mathrm{f} / \mathrm{day}(5 \mathrm{f} / \mathrm{qw})$ for $6-7$ weeks \\
\hline
\end{tabular}

Notes: a One hundred ninety-three patients were registered, but only 147 patients were analyzed. ${ }^{\mathrm{b}}$ The study by Kwong et al consisted of four treatment arms. Abbreviations: 2D-RT, two-dimensional radiotherapy; AUC, area under concentration-time curve; CRT, concurrent chemoradiotherapy; DDP, cisplatin; f, fraction; Fu, fluorouracil; IMRT, intensity-modulated radiotherapy; q3w, every 3 weeks; q4w, every 4 weeks; RT, radiotherapy. 


\begin{tabular}{|c|c|c|}
\hline \multicolumn{3}{|l|}{ Chemotherapy protocol } \\
\hline Induction phase & Concurrent phase & Adjuvant phase \\
\hline None & DDP $100 \mathrm{mg} / \mathrm{m}^{2} \mathrm{dl} \mathrm{q} 3 \mathrm{w} \times 3$ & $\begin{array}{l}\text { DDP } 80 \mathrm{mg} / \mathrm{m}^{2} \mathrm{dl}+\mathrm{Fu} \mathrm{I}, 000 \mathrm{mg} / \mathrm{m}^{2} / \text { day } \mathrm{dl}-4 \\
\text { civ q3 } \mathrm{w} \times 4\end{array}$ \\
\hline None & $\begin{array}{l}\text { UFT } 200 \mathrm{mg} \text { TID, } 7 \text { days per week for } \\
\text { 5-8 weeks }\end{array}$ & $\begin{array}{l}\text { DDP } 100 \mathrm{mg} / \mathrm{m}^{2} \mathrm{dl}+5-\mathrm{FU} \mathrm{I}, 000 \mathrm{mg} / \mathrm{m}^{2} / \mathrm{d} \mathrm{dl}-3 \\
\text { civ, } \mathrm{q} 3 \mathrm{w} \times 6 \\
\text { or vincristine } 2 \mathrm{mg} \mathrm{dl}+\text { bleomycin } 30 \mathrm{mg} \mathrm{dl}+ \\
\text { methotrexate } 150 \mathrm{mg} / \mathrm{m}^{2} \mathrm{dl}, \mathrm{q} 3 \mathrm{w} \times 6\end{array}$ \\
\hline None & $\begin{array}{l}\text { DDP } 25 \mathrm{mg} / \mathrm{m}^{2} / \text { day for } 4 \text { days or } 30 / 30 / 40 \\
\mathrm{mg} / \mathrm{m}^{2} / \text { day for } 3 \text { days } \mathrm{q} 3 \mathrm{w} \times 3\end{array}$ & $\begin{array}{l}\text { DDP } 20 \mathrm{mg} / \mathrm{m}^{2} / \text { day for } 4 \text { days }+ \text { Fu I, } 000 \mathrm{mg} / \\
\mathrm{m}^{2} / \text { day dI-4 q3w } \times 3\end{array}$ \\
\hline None & $100 \mathrm{mg} / \mathrm{m}^{2} \mathrm{dl} \mathrm{q} 3 \mathrm{w} \times 3$ & $\begin{array}{l}\text { DDP } 80 \mathrm{mg} / \mathrm{m}^{2} \mathrm{dl}+\mathrm{I}, 000 \mathrm{mg} / \mathrm{m}^{2} / \text { day } \mathrm{dl}-4 \text { civ } \\
\mathrm{q} 4 \mathrm{w} \times 3\end{array}$ \\
\hline None & $100 \mathrm{mg} / \mathrm{m}^{2} \mathrm{dl} \mathrm{q} 3 \mathrm{w} \times 3$ & $\begin{array}{l}\text { DDP } 80 \mathrm{mg} / \mathrm{m}^{2} \mathrm{dl}+\mathrm{I}, 000 \mathrm{mg} / \mathrm{m}^{2} / \text { day } \mathrm{dl}-4 \text { civ } \\
\mathrm{q} 4 \mathrm{w} \times 3\end{array}$ \\
\hline None & $100 \mathrm{mg} / \mathrm{m}^{2} \mathrm{dl} \mathrm{q} 3 \mathrm{w} \times 3$ & $\begin{array}{l}\text { DDP } 80 \mathrm{mg} / \mathrm{m}^{2} \mathrm{dl}+\mathrm{Fu} 800 \mathrm{mg} / \mathrm{m}^{2} / \text { day dl }-5 \\
\text { civ q } 3 \mathrm{w} \times 3\end{array}$ \\
\hline None & DDP $40 \mathrm{mg} / \mathrm{m}^{2} \mathrm{dl}$ weekly for 8 weeks & None \\
\hline None & $\begin{array}{l}\text { UFT } 200 \mathrm{mg} \text { TID, } 7 \text { days per week for } \\
\text { 5-8 weeks }\end{array}$ & None \\
\hline None & $\begin{array}{l}\text { Oxaliplatin } 70 \mathrm{mg} / \mathrm{m}^{2} \mathrm{dl} \text { weekly for } 6 \\
\text { weeks }\end{array}$ & None \\
\hline $\begin{array}{l}\text { Docetaxel } 75 \mathrm{mg} / \mathrm{m}^{2} \mathrm{dl}+\text { DDP } 75 \mathrm{mg} / \mathrm{m}^{2} \mathrm{dl} \\
\mathrm{q} 3 \mathrm{w} \times 2\end{array}$ & $40 \mathrm{mg} / \mathrm{m}^{2} \mathrm{dl} \mathrm{qw} \times 8$ & None \\
\hline $\begin{array}{l}\text { Paclitaxel } 70 \mathrm{mg} / \mathrm{m}^{2} \mathrm{dl}, \mathrm{d} 8+\text { Carboplatin AUC }=2.5 \\
\mathrm{dl}, \mathrm{d} 8+\text { Gemcitabine } \mathrm{I}, 000 \mathrm{mg} / \mathrm{m}^{2} \mathrm{dl}, \mathrm{d} 8 \mathrm{q} 3 \mathrm{w} \times 3\end{array}$ & DDP $40 \mathrm{mg} / \mathrm{m}^{2}$ weekly for 8 weeks & None \\
\hline $\begin{array}{l}\text { Docetaxel } 60 \mathrm{mg} / \mathrm{m}^{2} \mathrm{dl}+\text { DDP } 60 \mathrm{mg} / \mathrm{m}^{2} \mathrm{dl}+\mathrm{Fu} \\
600 \mathrm{mg} / \mathrm{m}^{2} / \text { day dl-5 civ q } 3 \mathrm{w} \times 3\end{array}$ & $100 \mathrm{mg} / \mathrm{m}^{2} \mathrm{dl} \mathrm{q} 3 \mathrm{w} \times 3$ & None \\
\hline $\begin{array}{l}\text { DDP } 80 \mathrm{mg} / \mathrm{m}^{2} \mathrm{dl}+\mathrm{Fu} 800 \mathrm{mg} / \mathrm{m}^{2} / \text { day dl }-5 \text { civ } \\
\text { q } 3 \mathrm{w} \times 3\end{array}$ & $80 \mathrm{mg} / \mathrm{m}^{2} \mathrm{dl} \mathrm{q} 3 \mathrm{w} \times 3$ & None \\
\hline $\begin{array}{l}\text { Docetaxel } 75 \mathrm{mg} / \mathrm{m}^{2} \mathrm{dl}+\text { DDP } 75 \mathrm{mg} / \mathrm{m}^{2} \mathrm{dl}+ \\
\text { 5-Fu } 750 \mathrm{mg} / \mathrm{m}^{2} / \text { day dI-d5 }\end{array}$ & DDP $40 \mathrm{mg} / \mathrm{m}^{2} /$ weekly & None \\
\hline $\begin{array}{l}\text { Mitomycin } 8 \mathrm{mg} / \mathrm{m}^{2} \mathrm{dl}+\text { epirubicin } 60 \mathrm{mg} / \mathrm{m}^{2} \\
\mathrm{dl}+\text { DDP } 60 \mathrm{mg} / \mathrm{m}^{2} \mathrm{dl}+5-\text { Fu } 450 \mathrm{mg} / \mathrm{m}^{2} \mathrm{~d} 8+ \\
\text { leucovorin } 30 \mathrm{mg} / \mathrm{m}^{2} \mathrm{~d} 8\end{array}$ & DDP $30 \mathrm{mg} / \mathrm{m}^{2} /$ weekly & None \\
\hline None & $\begin{array}{l}\text { UFT } 200 \mathrm{mg} \text { TID, } 7 \text { days per week for } \\
\text { 5-8 weeks }\end{array}$ & $\begin{array}{l}\text { DDP } 100 \mathrm{mg} / \mathrm{m}^{2} \mathrm{dl}+5-\mathrm{FU} \mathrm{I}, 000 \mathrm{mg} / \mathrm{m}^{2} / \mathrm{d} \mathrm{dl}-3 \\
\text { civ, q3w } \times 6 \\
\text { or vincristine } 2 \mathrm{mg} \mathrm{dl}+\text { bleomycin } 30 \mathrm{mg} \mathrm{dl}+ \\
\text { methotrexate } 150 \mathrm{mg} / \mathrm{m}^{2} \mathrm{dl}, \mathrm{q} 3 \mathrm{w} \times 6\end{array}$ \\
\hline None & $\begin{array}{l}\text { DDP } 40 \mathrm{mg} / \mathrm{m}^{2} \mathrm{dl} \text { weekly for up to } 7 \\
\text { weeks }\end{array}$ & $\begin{array}{l}\text { DDP } 80 \mathrm{mg} / \mathrm{m}^{2} \mathrm{dl}+\mathrm{Fu} 800 \mathrm{mg} / \mathrm{m}^{2} / \text { day dI-5 } \\
\text { civ } \mathrm{q} 4 \mathrm{w} \times 3\end{array}$ \\
\hline
\end{tabular}




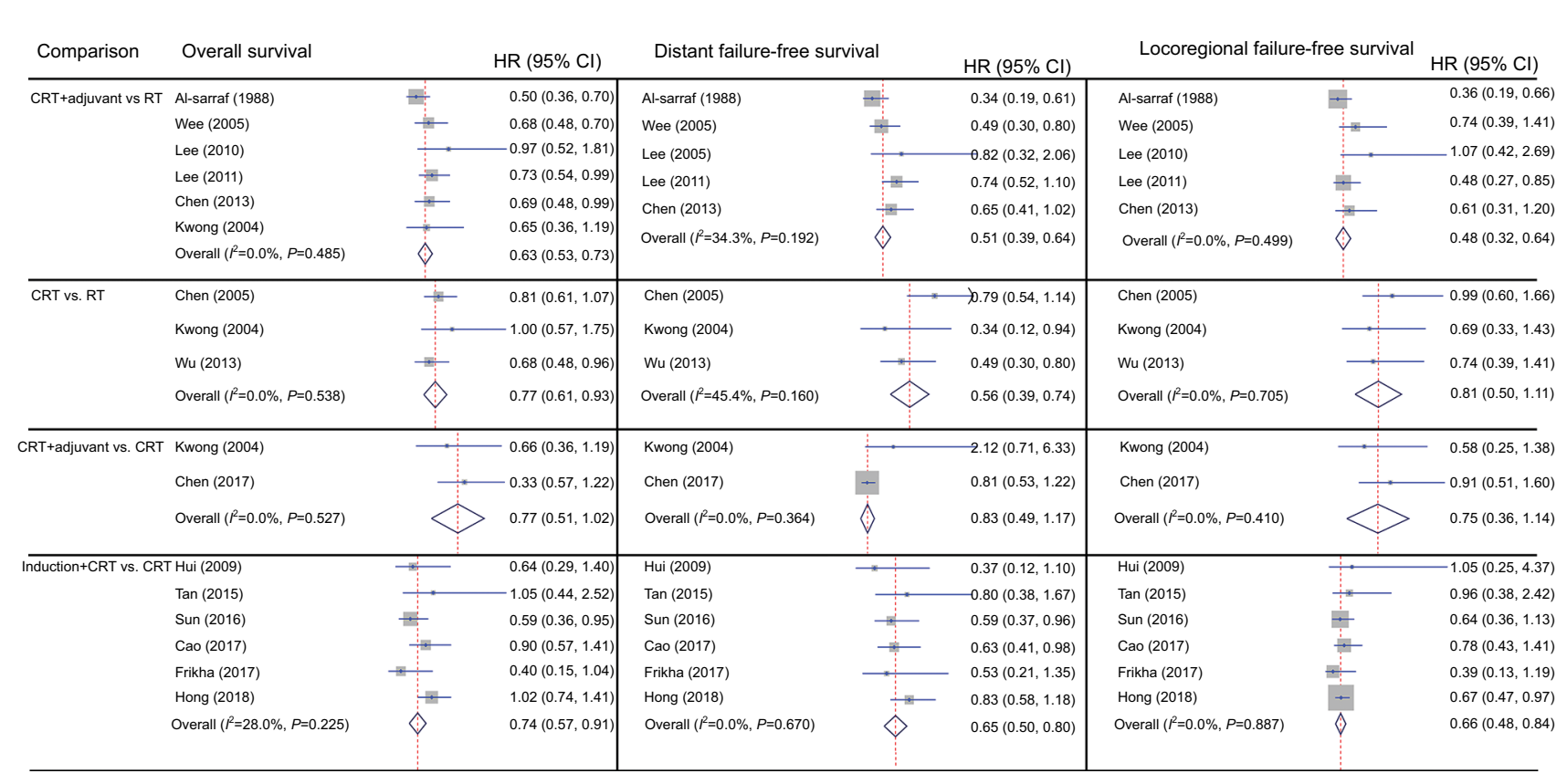

Figure I Forest plot of pairwise meta-analysis.

Abbreviations: civ, continuous intravenous injection; CRT, concurrent chemoradiotherapy; RT, radiotherapy; UFT, uracil-FT-207.

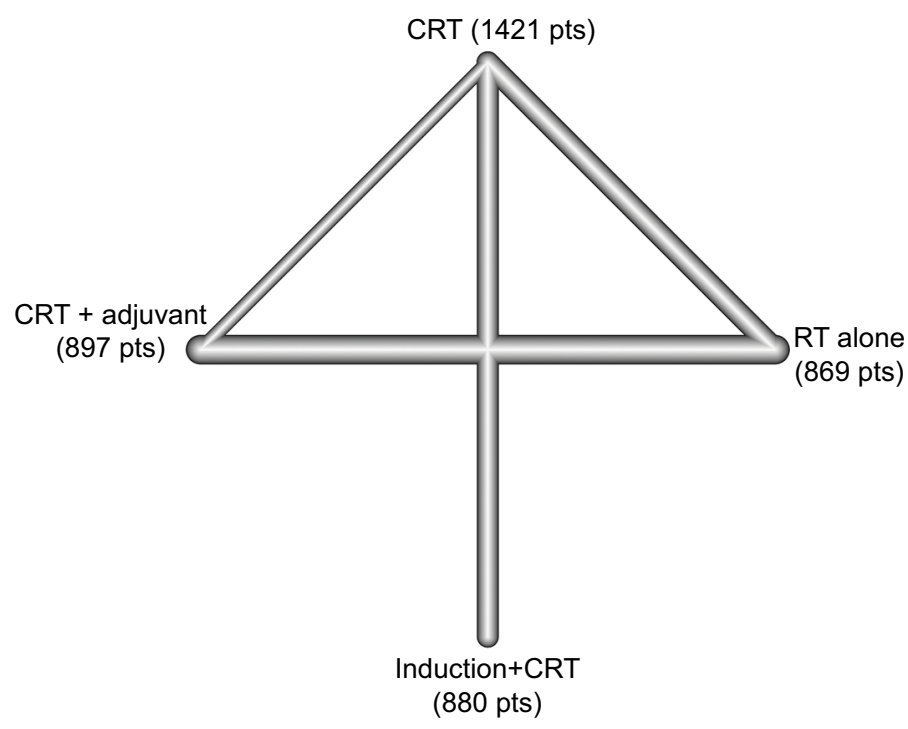

Figure 2 Graph of the network comparisons for overall survival.

Abbreviations: CRT, concurrent chemoradiotherapy; RT, radiotherapy.

alone was worse than the other three treatments. Undoubtedly, induction + CRT achieved highest P-score of 98.6\% (Table 2), indicating that induction + CRT may be the best choice for reducing distant failure.

With regard to LFFS, induction + CRT was better than CRT (HR, 0.69; 95\% CI, 0.54-0.89) and RT alone (HR, $0.55 ; 95 \%$ CI, 0.37-0.81). Moreover, CRT + adjuvant was also better than RT (HR, 0.58; 95\% CI, 0.44-0.75). Otherwise, no significant difference was identified between other comparisons. Similarly, induction + CRT still achieved the highest P-score of $86.2 \%$ (Table 2).

Given these findings, CRT + adjuvant may be a better treatment for improving OS, while induction + CRT was better in reducing distant failure and locoregional failure.

\section{Sensitivity analysis}

To validate the stability of our findings, we performed sensitivity analysis after excluding the study by Kwong et $\mathrm{al}^{38}$ 
Table 2 Results of network multiple comparisons

\begin{tabular}{|l|l|l|l|}
\hline Treatment arms & OS & DFFS & LRFS \\
\hline P-value of total heterogeneity/inconsistency & $0.5 \mathrm{I}$ & 0.27 & 0.79 \\
P-value of within-study heterogeneity & 0.44 & 0.21 & 0.75 \\
$\begin{array}{l}\text { P-value of between-study heterogeneity } \\
\text { CRT (reference group) }\end{array}$ & 0.69 & 0.94 & 0.62 \\
$\quad$ Hazard ratio & 1.00 & 1.00 & 1.00 \\
$\quad$ P-score & $35.3 \%$ & $42.7 \%$ & $32.1 \%$ \\
CRT + adjuvant & $0.82(0.67-1.00)$ & $0.94(0.70-1.28)$ & $0.73(0.52-1.01)$ \\
Hazard ratio $(95 \% \mathrm{Cl})$ & $84.1 \%$ & $58.7 \%$ & $79.4 \%$ \\
P-score & $0.83(0.67-1.03)$ & $0.67(0.53-0.86)$ & $0.69(0.54-0.89)$ \\
Induction + CRT & $80.3 \%$ & $98.6 \%$ & $86.2 \%$ \\
$\quad$ Hazard ratio $(95 \% \mathrm{Cl})$ & $1.25(1.05-1.49)$ & $1.63(1.23-2.14)$ & $1.25(0.93-1.69)$ \\
P-score & $0.3 \%$ & $<0.1 \%$ & $2.3 \%$ \\
RT alone & & \\
Hazard ratio $(95 \% \mathrm{Cl})$ & & \\
P-score & &
\end{tabular}

Notes: Fixed-effects model was used for OS, DFFS, and locoregional failure-free survival.

Abbreviations: CRT, concurrent chemoradiotherapy; DFFS, distant failure-free survival; LRFS, locoregional recurrence-free survival; OS, overall survival.
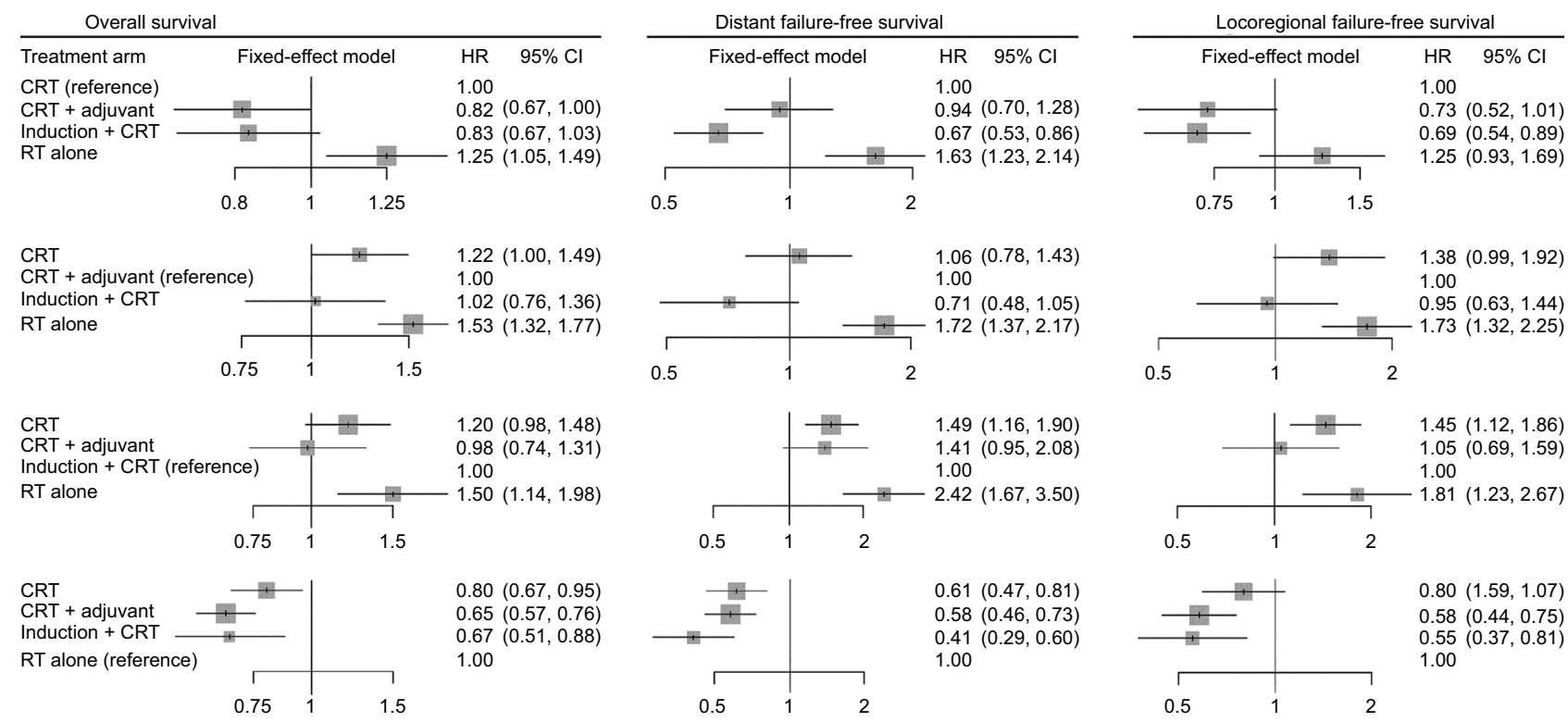

$0.80(1.59,1.07)$ $0.58(0.44,0.75)$ $0.55(0.37,0.81)$ 1.00

Figure 3 Forest plot of network meta-analysis.

Abbreviations: CRT, concurrent chemoradiotherapy; RT, radiotherapy.

because the chemotherapeutic agent used during RT is not platinum. The network plot was shown in Figure S2. As indicated by the results (Figure 4, Table 3), induction + CRT was still better than CRT in terms of DFFS (HR, 0.68; 95\% CI, 0.54-0.85) and LFFS (HR, 0.69; 95\% CI, 0.54-0.89), while no significant difference between CRT + adjuvant and CRT, or between induction + CRT and CRT + adjuvant was found. RT alone was still worse than the other treatments. Intriguingly, induction + CRT achieved the highest P-score on OS, DFFS, and LFFS. Taken these together, our results remained valid in sensitivity analysis.

\section{Discussion}

Our current network meta-analysis compared four treatment arms (CRT, CRT + adjuvant, induction + CRT, and RT alone) in advanced NPC and found that induction + CRT was better than CRT in reducing distant metastasis and locoregional recurrence, while CRT + adjuvant was superior to CRT in improving OS. Notably, induction + CRT did not significantly differ from CRT + adjuvant. RT alone was always worse than the other three treatments. Further, sensitivity analysis after excluding the study by Kwong et $\mathrm{al}^{38}$ also confirmed these findings. 

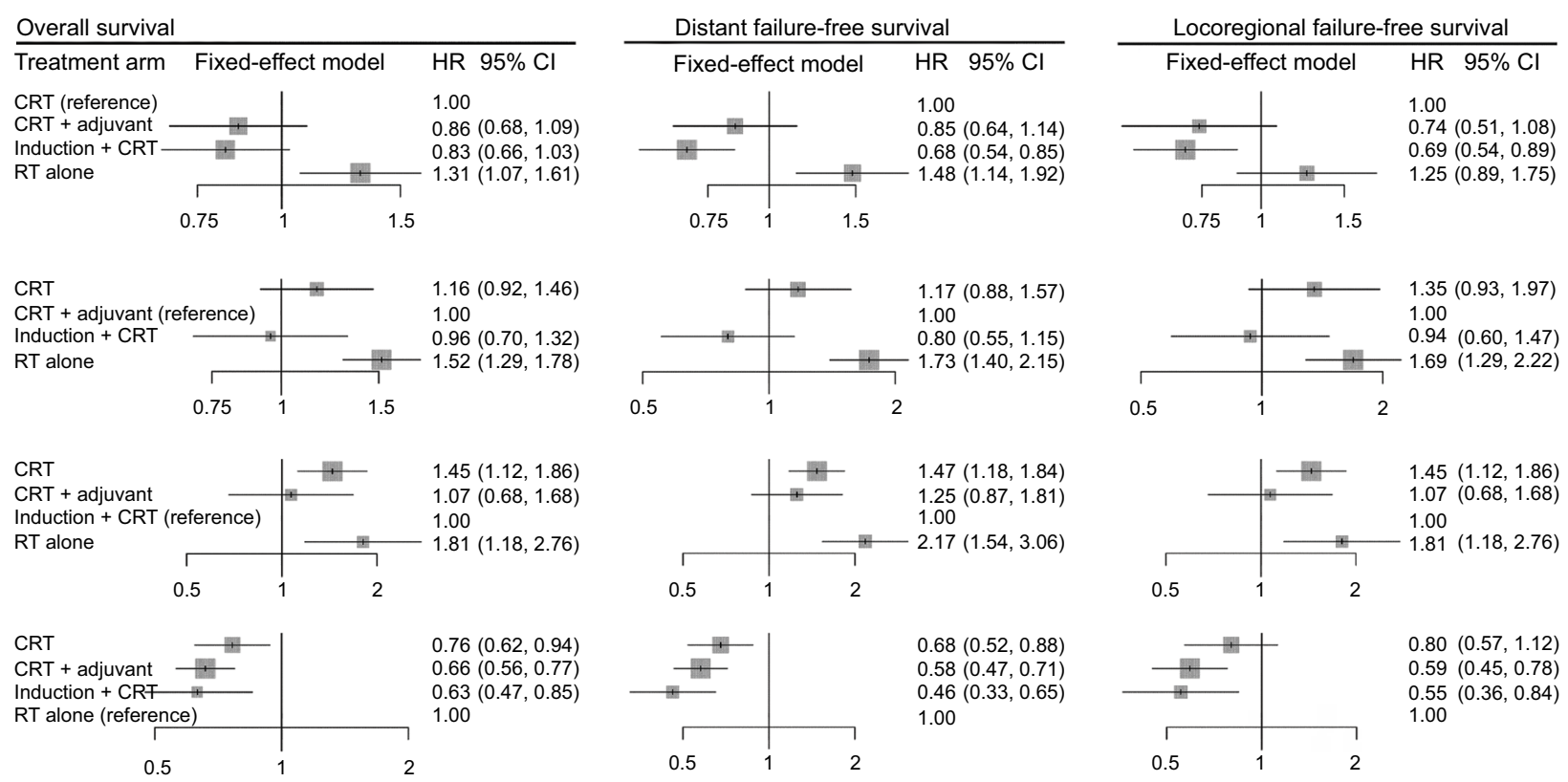

Figure 4 Forest plot of sensitivity analysis.

Abbreviations: CRT, concurrent chemoradiotherapy; RT, radiotherapy.

Table 3 Results of network multiple comparisons after excluding the study by Kwong et al ${ }^{38}$

\begin{tabular}{|l|l|l|l|}
\hline Treatment arms & OS & DFFS & LRFS \\
\hline P-value of total heterogeneity/inconsistency & 0.37 & 0.40 & 0.69 \\
P-value of within-study heterogeneity & 0.30 & 0.32 & 0.69 \\
P-value of between-study heterogeneity & 0.82 & 0.75 & 0.35 \\
CRT (reference group) & 1.00 & 1.00 & 1.00 \\
$\quad$ Hazard ratio & $37.7 \%$ & $37.6 \%$ & $32.0 \%$ \\
P-score & & & $0.74(0.51-1.08)$ \\
CRT + adjuvant & $0.86(0.68-1.09)$ & $0.85(0.64-1.14)$ & $77.6 \%$ \\
Hazard ratio $(95 \% \mathrm{Cl})$ & $77.3 \%$ & $66.1 \%$ & $0.69(0.54-0.89)$ \\
P-score & & $0.68(0.54-0.85)$ & $87.0 \%$ \\
Induction + CRT & $0.83(0.66-1.03)$ & $96.3 \%$ & $1.25(0.89-1.75)$ \\
Hazard ratio $(95 \% \mathrm{Cl})$ & $84.8 \%$ & $1.48(1.14-1.92)$ & $3.4 \%$ \\
$\quad$ P-score & $1.31(1.07-1.6 \mathrm{I})$ & $<0.1 \%$ & \\
RT alone & $0.1 \%$ & \\
Hazard ratio $(95 \% \mathrm{Cl})$ & & \\
P-score & & \\
\hline
\end{tabular}

Notes: Fixed-effects model was used for OS, DFFS, and locoregional failure-free survival.

Abbreviations: CRT, concurrent chemoradiotherapy; DFFS, distant failure-free survival; LRFS, locoregional recurrence-free survival; OS, overall survival.

As intensity-modulated radiotherapy has become the predominant treatment for patients with NPC, locoregional control of advanced disease has improved greatly and distant metastasis has become the main failure pattern. ${ }^{41,42}$ Therefore, combined strategies of chemotherapy with radiotherapy have been intensively investigated. A possible and feasible strategy is additional cycles of chemotherapy, such as adjuvant chemotherapy and induction chemotherapy, to CRT. In fact, both $\mathrm{CRT}+$ adjuvant and induction + CRT have been recommended as the standard cure for patients with advanced NPC by the National Comprehensive Cancer Network (NCCN) guidelines. However, this produces another question: which one is better? CRT + adjuvant or induction + CRT? As far as we know, there are no head-to-head clinical trials comparing CRT + adjuvant with induction + CRT being reported yet. Given this concern, we performed this network meta-analysis to provide preliminary answer for this question. Our findings indicated that CRT + adjuvant and induction + CRT were equally effective. 
A recent individual patient data pooled analysis of four randomized clinical trials comparing induction + CRT with CRT revealed that induction + CRT was superior over CRT in reducing distant metastasis and improving OS. ${ }^{17}$ Similar to these findings, our study also demonstrated that induction + CRT was better than CRT both in direct and network meta-analysis. However, when comparing induction + CRT with CRT + adjuvant, no significant difference was found in both original and sensitivity analysis for all endpoints, which was consistent with the findings in an individual patient data network meta-analysis. ${ }^{15}$ Obviously, the incorporation of new evidence ${ }^{11,12,14}$ about induction + CRT into the network metaanalysis still failed to demonstrate the superiority of induction + CRT over CRT + adjuvant. One possible explanation is that previous value regarding CRT + adjuvant is too strong since RT alone was the control arm, ${ }^{47,39}$ therefore increasing the weight of CRT + adjuvant in the network comparison. Given these findings and concerns, head-to-head randomized clinical trials comparing induction + CRT with CRT + adjuvant are urgently warranted. Possibly, only results from such trials could be conclusive.

To validate the results of original analysis, we performed sensitivity analysis after excluding the study by Kwong et $a{ }^{38}$ because a standard concurrent chemotherapy regimen of cisplatin was not used in that trial. Generally, the results of sensitivity remained valid, indicating that our meta-analysis was reliable. Of note, CRT + adjuvant was better than CRT for OS in original analysis, while they were comparable in sensitivity analysis. The reason being only one trial comparing CRT + adjuvant with CRT existed ${ }^{8}$ after excluding the study by Kwong et al. ${ }^{38}$ Actually, no significant difference between CRT + adjuvant and CRT was found in the original text. ${ }^{8}$ Undoubtedly, a lack of such trials would affect the final results of network meta-analysis. Therefore, future metaanalysis should include more trials.

It should be pointed out that we could not only rely on the P-score to select treatment although we employed it to rank treatment. A treatment ranking probability would still be generated without definitive statistical significance even if differences of effect size between treatments were small and nonsignificant. Thus, we should not overinterpret the P-score in network meta-analysis.

We should also address the limitations of this study. Since we have no access to individual patient data, potentially reporting bias could be produced. To minimize such bias, we set strict criteria for study inclusion to reduce heterogeneity between studies. Another important issue should be the application of different radiotherapy technique (intensity- modulated radiotherapy vs conventional radiotherapy) across different trials which may affect the results. Moreover, we did not evaluate the endpoint of PFS because the definition of PFS varied greatly across trials. Of course, these limitations should be addressed in future studies.

\section{Conclusion}

Overall, our current study showed the indirect results of comparing CRT + adjuvant with induction + CRT in locoregionally advanced NPC and found that they were equally effective but both better than CRT alone. Future head-to-head clinical trials were needed to provide more conclusive evidence for optimal treatment strategy in advanced NPC.

\section{Disclosure}

The authors report no conflicts of interest in this work.

\section{References}

1. Jemal A, Bray F, Center MM, Ferlay J, Ward E, Forman D. Global cancer statistics. CA Cancer J Clin. 2011;61(2):69-90.

2. Chen W, Zheng R, Baade PD, et al. Cancer statistics in China, 2015. CA Cancer J Clin. 2016;66(2):115-132.

3. Wei KR, Zheng RS, Zhang SW, Liang ZH, Li ZM, Chen WQ. Nasopharyngeal carcinoma incidence and mortality in China, 2013. Chin J Cancer. 2017;36(1):90.

4. Al-Sarraf M, Leblanc M, Giri PG, et al. Chemoradiotherapy versus radiotherapy in patients with advanced nasopharyngeal cancer: phase III randomized Intergroup study 0099. J Clin Oncol. 1998;16(4): 1310-1317.

5. Chen Y, Sun Y, Liang SB, et al. Progress report of a randomized trial comparing long-term survival and late toxicity of concurrent chemoradiotherapy with adjuvant chemotherapy versus radiotherapy alone in patients with stage III to IVB nasopharyngeal carcinoma from endemic regions of China. Cancer. 2013;119(12):2230-2238.

6. Lee AW, Tung SY, Chua DT, et al. Randomized trial of radiotherapy plus concurrent-adjuvant chemotherapy vs radiotherapy alone for regionally advanced nasopharyngeal carcinoma. $J$ Natl Cancer Inst. 2010;102(15):1188-1198.

7. Wee J, Tan EH, Tai BC, et al. Randomized trial of radiotherapy versus concurrent chemoradiotherapy followed by adjuvant chemotherapy in patients with American Joint Committee on Cancer/International Union against cancer stage III and IV nasopharyngeal cancer of the endemic variety. J Clin Oncol. 2005;23(27):6730-6738.

8. Chen L, Hu CS, Chen XZ, et al. Adjuvant chemotherapy in patients with locoregionally advanced nasopharyngeal carcinoma: Long-term results of a phase 3 multicentre randomised controlled trial. Eur J Cancer. 2017;75:150-158.

9. Yi JL, Gao L, Huang XD, et al. Nasopharyngeal carcinoma treated by radical radiotherapy alone: ten-year experience of a single institution. Int J Radiat Oncol Biol Phys. 2006;65(1):161-168.

10. Cao SM, Yang Q, Guo L, et al. Neoadjuvant chemotherapy followed by concurrent chemoradiotherapy versus concurrent chemoradiotherapy alone in locoregionally advanced nasopharyngeal carcinoma: a phase III multicentre randomised controlled trial. Eur J Cancer. 2017;75: 14-23.

11. Sun Y, Li WF, Chen NY, et al. Induction chemotherapy plus concurrent chemoradiotherapy versus concurrent chemoradiotherapy alone in locoregionally advanced nasopharyngeal carcinoma: a phase 3, multicentre, randomised controlled trial. Lancet Oncol. 2016;17(11):1509-1520. 
12. Frikha M, Auperin A, Tao Y, et al. A randomized trial of induction docetaxel-cisplatin-5FU followed by concomitant cisplatin-RT versus concomitant cisplatin-RT in nasopharyngeal carcinoma (GORTEC 2006-02). Ann Oncol. 2018;29(3):731-736.

13. Hui EP, Ma BB, Leung SF, et al. Randomized phase II trial of concurrent cisplatin-radiotherapy with or without neoadjuvant docetaxel and cisplatin in advanced nasopharyngeal carcinoma. J Clin Oncol. 2009;27(2):242-249.

14. Hong RL, Hsiao CF, Ting LL, et al. Final results of a randomized phase III trial of induction chemotherapy followed by concurrent chemoradiotherapy versus concurrent chemoradiotherapy alone in patients with stage IVA and IVB nasopharyngeal carcinoma-Taiwan Cooperative Oncology Group (TCOG) 1303 Study. Ann Oncol. 2018;29(9):1972-1979.

15. Ribassin-Majed L, Marguet S, Lee AWM, et al. What is the best treatment of locally advanced nasopharyngeal carcinoma? An individual patient data network meta-analysis. J Clin Oncol. 2017;35(5):498-505.

16. Jadad AR, Moore RA, Carroll D, et al. Assessing the quality of reports of randomized clinical trials: is blinding necessary? Control Clin Trials. 1996;17(1):1-12.

17. Chen YP, Tang LL, Yang Q, et al. Induction chemotherapy plus concurrent chemoradiotherapy in endemic nasopharyngeal carcinoma: individual patient data pooled analysis of four randomized trials. Clin Cancer Res. 2018;24(8):1824-1833.

18. Rücker G, Schwarzer G. Ranking treatments in frequentist network meta-analysis works without resampling methods. BMC Med Res Methodol. 2015;15:58.

19. Rücker G. Network meta-analysis, electrical networks and graph theory. Res Synth Methods. 2012;3(4):312-324.

20. Chaimani A, Higgins JP, Mavridis D, Spyridonos P, Salanti G. Graphical tools for network meta-analysis in STATA. PLoS One. 2013;8(10):e76654.

21. Salanti G, Ades AE, Ioannidis JP. Graphical methods and numerical summaries for presenting results from multiple-treatment meta-analysis: an overview and tutorial. J Clin Epidemiol. 2011;64(2):163-171.

22. Lin JC, Jan JS, Hsu CY, Liang WM, Jiang RS, Wang WY. Phase III study of concurrent chemoradiotherapy versus radiotherapy alone for advanced nasopharyngeal carcinoma: positive effect on overall and progression-free survival. J Clin Oncol. 2003;21(4):631-637.

23. Chen QY, Wen YF, Guo L, et al. Concurrent chemoradiotherapy vs radiotherapy alone in stage II nasopharyngeal carcinoma: phase III randomized trial. J Natl Cancer Inst. 2011;103(23):1761-1770.

24. Fountzilas G, Ciuleanu E, Bobos M, et al. Induction chemotherapy followed by concomitant radiotherapy and weekly cisplatin versus the same concomitant chemoradiotherapy in patients with nasopharyngeal carcinoma: a randomized phase II study conducted by the Hellenic Cooperative Oncology Group (HeCOG) with biomarker evaluation. Ann Oncol. 2012;23(2):427-435.

25. Lee AW, Ngan RK, Tung SY, et al. Preliminary results of trial NPC-0501 evaluating the therapeutic gain by changing from concurrent-adjuvant to induction-concurrent chemoradiotherapy, changing from fluorouracil to capecitabine, and changing from conventional to accelerated radiotherapy fractionation in patients with locoregionally advanced nasopharyngeal carcinoma. Cancer. 2015;121(8):1328-1338.

26. Lee AW, Tung SY, Chan AT, et al. A randomized trial on addition of concurrent-adjuvant chemotherapy and/or accelerated fractionation for locally-advanced nasopharyngeal carcinoma. Radiother Oncol. 2011;98(1):15-22.

27. Lee AW, Tung SY, Chan AT, et al. Preliminary results of a randomized study (NPC-9902 Trial) on therapeutic gain by concurrent chemotherapy and/or accelerated fractionation for locally advanced nasopharyngeal carcinoma. Int J Radiat Oncol Biol Phys. 2006;66(1):142-151.
28. Chen L, Hu CS, Chen XZ, et al. Concurrent chemoradiotherapy plus adjuvant chemotherapy versus concurrent chemoradiotherapy alone in patients with locoregionally advanced nasopharyngeal carcinoma: a phase $3 \mathrm{mul}-$ ticentre randomised controlled trial. Lancet Oncol. 2012;13(2):163-171.

29. Chen L, Hu CS, Chen XZ, et al. Adjuvant chemotherapy in patients with locoregionally advanced nasopharyngeal carcinoma: Long-term results of a phase 3 multicentre randomised controlled trial. Eur J Cancer. 2017;75:150-158.

30. Chan AT, Leung SF, Ngan RK, et al. Overall survival after concurrent cisplatin-radiotherapy compared with radiotherapy alone in locoregionally advanced nasopharyngeal carcinoma. J Natl Cancer Inst. 2005;97(7):536-539.

31. Chan AT, Teo PM, Ngan RK, et al. Concurrent chemotherapy-radiotherapy compared with radiotherapy alone in locoregionally advanced nasopharyngeal carcinoma: progression-free survival analysis of a phase III randomized trial. J Clin Oncol. 2002;20(8):2038-2044.

32. Lee AW, Lau WH, Tung SY, et al. Preliminary results of a randomized study on therapeutic gain by concurrent chemotherapy for regionally-advanced nasopharyngeal carcinoma: NPC-9901 Trial by the Hong Kong Nasopharyngeal Cancer Study Group. J Clin Oncol. 2005;23(28):6966-6975.

33. Chen Y, Liu MZ, Liang SB, et al. Preliminary results of a prospective randomized trial comparing concurrent chemoradiotherapy plus adjuvant chemotherapy with radiotherapy alone in patients with locoregionally advanced nasopharyngeal carcinoma in endemic regions of China. Int J Radiat Oncol Biol Phys. 2008;71(5):1356-1364.

34. Chen Y, Sun Y, Liang SB, et al. Progress report of a randomized trial comparing long-term survival and late toxicity of concurrent chemoradiotherapy with adjuvant chemotherapy versus radiotherapy alone in patients with stage III to IVB nasopharyngeal carcinoma from endemic regions of China. Cancer. 2013;119(12):2230-2238.

35. Wu X, Huang PY, Peng PJ, et al. Long-term follow-up of a phase III study comparing radiotherapy with or without weekly oxaliplatin for locoregionally advanced nasopharyngeal carcinoma. Ann Oncol. 2013;24(8):2131-2136.

36. Zhang L, Zhao C, Peng PJ, et al. Phase III study comparing standard radiotherapy with or without weekly oxaliplatin in treatment of locoregionally advanced nasopharyngeal carcinoma: preliminary results. J Clin Oncol. 2005;23(33):8461-8468.

37. Chan AT, Leung SF, Ngan RK, et al. Overall survival after concurrent cisplatin-radiotherapy compared with radiotherapy alone in locoregionally advanced nasopharyngeal carcinoma. J Natl Cancer Inst. 2005;97(7):536-539.

38. Kwong DL, Sham JS, Au GK, et al. Concurrent and adjuvant chemotherapy for nasopharyngeal carcinoma: a factorial study. J Clin Oncol. 2004;22(13):2643-2653.

39. Lee AW, Tung SY, Chan AT, et al. A randomized trial on addition of concurrent-adjuvant chemotherapy and/or accelerated fractionation for locally-advanced nasopharyngeal carcinoma. Radiother Oncol. 2011;98(1):15-22.

40. Tan T, Lim WT, Fong KW, et al. Concurrent chemo-radiation with or without induction gemcitabine, Carboplatin, and Paclitaxel: a randomized, phase $2 / 3$ trial in locally advanced nasopharyngeal carcinoma. Int J Radiat Oncol Biol Phys. 2015;91(5):952-960.

41. Lai SZ, Li WF, Chen L, et al. How does intensity-modulated radiotherapy versus conventional two-dimensional radiotherapy influence the treatment results in nasopharyngeal carcinoma patients? Int J Radiat Oncol Biol Phys. 2011;80(3):661-668.

42. Sun X, Su S, Chen C, et al. Long-term outcomes of intensity-modulated radiotherapy for 868 patients with nasopharyngeal carcinoma: an analysis of survival and treatment toxicities. Radiother Oncol. 2014;110(3):398-403. 


\section{Supplementary materials}

Table SI Jadad/Oxford score for the 15 clinical trials

\begin{tabular}{|c|c|c|c|c|c|}
\hline Clinical trial & $\begin{array}{l}\text { Randomization } \\
\text { method }\end{array}$ & $\begin{array}{l}\text { Allocation } \\
\text { concealment }\end{array}$ & $\begin{array}{l}\text { Blinding } \\
\text { principle }\end{array}$ & $\begin{array}{l}\text { Patient } \\
\text { dropout }\end{array}$ & $\begin{array}{l}\text { Jadad } \\
\text { score }\end{array}$ \\
\hline \multicolumn{6}{|l|}{ CRT + adjuvant vs RT } \\
\hline Al-Sarraf et al (1998)' & No & No & No & Yes & 1 \\
\hline Kwong et al $(2004)^{2}$ & Yes & No & No & Yes & 3 \\
\hline Wee et al $(2005)^{3}$ & Yes & Yes & No & Yes & 4 \\
\hline Lee et al $(2010)^{4}$ & Yes & Yes & No & Yes & 4 \\
\hline Lee et al $(20 \mathrm{II})^{5}$ & Yes & Yes & No & Yes & 4 \\
\hline Chen et al $(2013)^{6}$ & Yes & Yes & Yes & Yes & 6 \\
\hline \multicolumn{6}{|l|}{ CRT vs RT } \\
\hline Chan et al $(2005)^{7}$ & Yes & Yes & No & Yes & 5 \\
\hline Kwong et al $(2004)^{2}$ & Yes & No & No & Yes & 3 \\
\hline Zhang et al $(2005)^{8}$ & No & No & No & Yes & 1 \\
\hline \multicolumn{6}{|l|}{ Induction + CRT vs CRT } \\
\hline Hui et al (2009)9 & Yes & Yes & No & Yes & 5 \\
\hline Tan et al $(2015)^{10}$ & Yes & Yes & No & Yes & 4 \\
\hline Sun et al $(2016)^{11}$ & Yes & Yes & No & Yes & 5 \\
\hline Cao et al $(2017)^{12}$ & Yes & Yes & No & Yes & 5 \\
\hline Frikha et al $(2018)^{13}$ & Yes & Yes & No & Yes & 5 \\
\hline Hong et al $(2018)^{14}$ & Yes & Yes & No & Yes & 5 \\
\hline \multicolumn{6}{|l|}{ CRT + adjuvant vs CRT } \\
\hline Kwong et al $(2004)^{2}$ & Yes & No & No & Yes & 3 \\
\hline Chen et al $(2017)^{15}$ & Yes & Yes & No & Yes & 5 \\
\hline
\end{tabular}

Abbreviations: CRT, concurrent chemoradiotherapy; RT, radiotherapy.
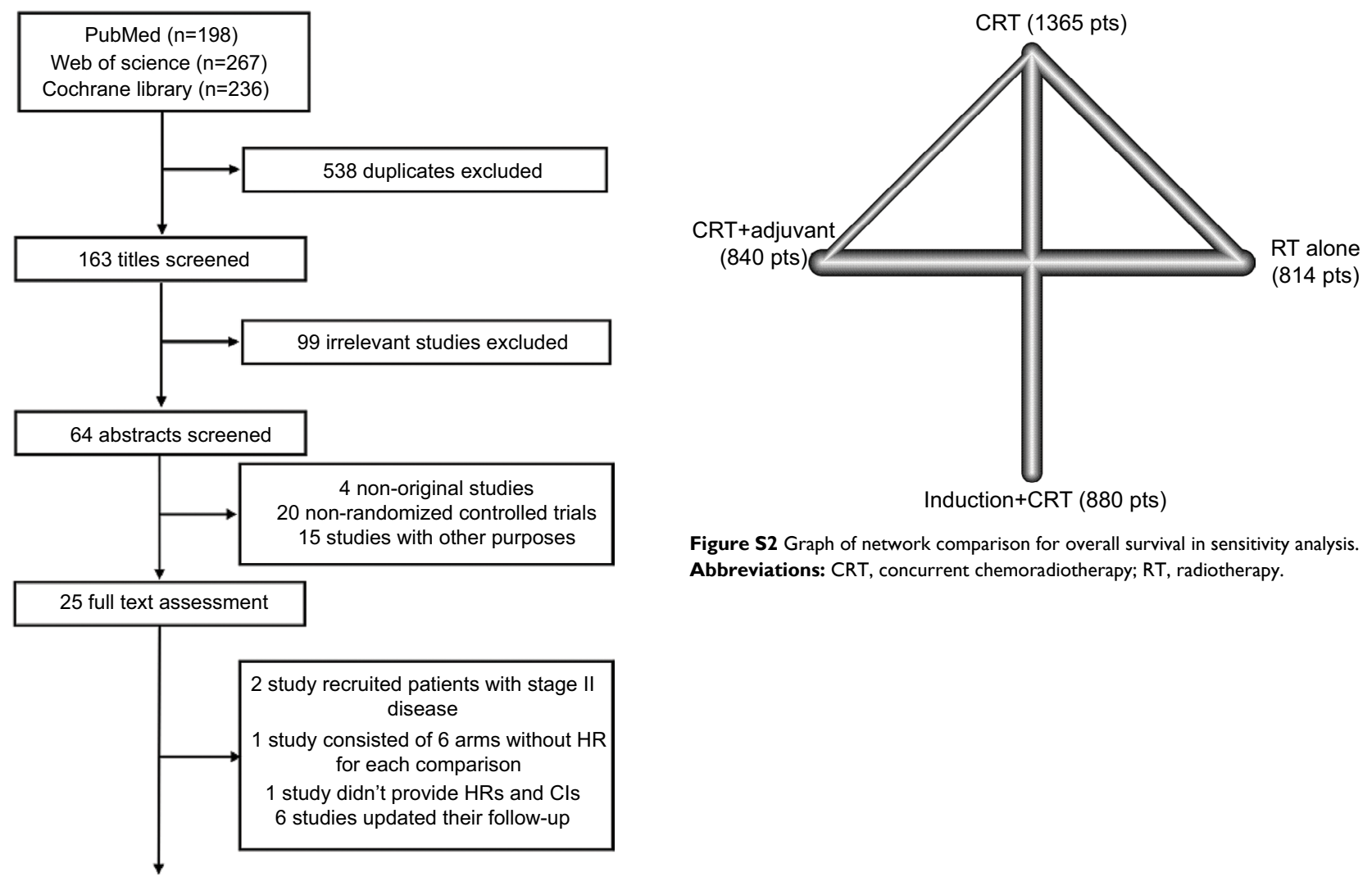

Figure S2 Graph of network comparison for overall survival in sensitivity analysis. Abbreviations: CRT, concurrent chemoradiotherapy; RT, radiotherapy.

15 studies eligible for this network meta-analysis

Figure SI Flowchart of study inclusion. 


\section{References}

1. Al-Sarraf M, Leblanc M, Giri PG, et al. Chemoradiotherapy versus radiotherapy in patients with advanced nasopharyngeal cancer: phase III randomized Intergroup study 0099. J Clin Oncol. 1998;16(4):1310-1317.

2. Kwong DL, Sham JS, Au GK, et al. Concurrent and adjuvant chemotherapy for nasopharyngeal carcinoma: a factorial study. J Clin Oncol. 2004;22(13):2643-2653.

3. Wee J, Tan EH, Tai BC, et al. Randomized trial of radiotherapy versus concurrent chemoradiotherapy followed by adjuvant chemotherapy in patients with American Joint Committee on Cancer/International Union against cancer stage III and IV nasopharyngeal cancer of the endemic variety. J Clin Oncol. 2005;23(27):6730-6738.

4. Lee AW, Tung SY, Chua DT, et al. Randomized trial of radiotherapy plus concurrent-adjuvant chemotherapy vs radiotherapy alone for regionally advanced nasopharyngeal carcinoma. J Natl Cancer Inst. 2010;102(15):1188-1198.

5. Lee AW, Tung SY, Chan AT, et al. A randomized trial on addition of concurrent-adjuvant chemotherapy and/or accelerated fractionation for locally-advanced nasopharyngeal carcinoma. Radiother Oncol. 2011;98(1):15-22.

6. Chen Y, Sun Y, Liang SB, et al. Progress report of a randomized trial comparing long-term survival and late toxicity of concurrent chemoradiotherapy with adjuvant chemotherapy versus radiotherapy alone in patients with stage III to IVB nasopharyngeal carcinoma from endemic regions of China. Cancer. 2013;119(12):2230-2238.

7. Chan AT, Leung SF, Ngan RK, et al. Overall survival after concurrent cisplatin-radiotherapy compared with radiotherapy alone in locoregionally advanced nasopharyngeal carcinoma. J Natl Cancer Inst. 2005;97(7):536-539.

8. Zhang L, Zhao C, Peng PJ, et al. Phase III study comparing standard radiotherapy with or without weekly oxaliplatin in treatment of locoregionally advanced nasopharyngeal carcinoma: preliminary results. J Clin Oncol. 2005;23(33):8461-8468.
9. Hui EP, Ma BB, Leung SF, et al. Randomized phase II trial of concurrent cisplatin-radiotherapy with or without neoadjuvant docetaxel and cisplatin in advanced nasopharyngeal carcinoma. J Clin Oncol. 2009;27(2):242-249.

10. Tan T, Lim WT, Fong KW, et al. Concurrent chemo-radiation with or without induction gemcitabine, Carboplatin, and Paclitaxel: a randomized, phase $2 / 3$ trial in locally advanced nasopharyngeal carcinoma. Int J Radiat Oncol Biol Phys. 2015;91(5):952-960.

11. Sun Y, Li WF, Chen NY, et al. Induction chemotherapy plus concurrent chemoradiotherapy versus concurrent chemoradiotherapy alone in locoregionally advanced nasopharyngeal carcinoma: a phase 3, multicentre, randomised controlled trial. Lancet Oncol. 2016;17(11):1509-1520.

12. Cao SM, Yang Q, Guo L, et al. Neoadjuvant chemotherapy followed by concurrent chemoradiotherapy versus concurrent chemoradiotherapy alone in locoregionally advanced nasopharyngeal carcinoma: a phase III multicentre randomised controlled trial. Eur J Cancer. 2017;75: 14-23.

13. Frikha M, Auperin A, Tao Y, et al. A randomized trial of induction docetaxel-cisplatin-5FU followed by concomitant cisplatin-RT versus concomitant cisplatin-RT in nasopharyngeal carcinoma (GORTEC 2006-02). Ann Oncol. 2018;29(3):731-736.

14. Hong RL, Hsiao CF, Ting LL, et al. Final results of a randomized phase III trial of induction chemotherapy followed by concurrent chemoradiotherapy versus concurrent chemoradiotherapy alone in patients with stage IVA and IVB nasopharyngeal carcinoma-Taiwan Cooperative Oncology Group (TCOG) 1303 Study. Ann Oncol. 2018;29(9):1972-1979.

15. Chen L, Hu CS, Chen XZ, et al. Adjuvant chemotherapy in patients with locoregionally advanced nasopharyngeal carcinoma: long-term results of a phase 3 multicentre randomised controlled trial. Eur J Cancer. 2017;75:150-158.
Cancer Management and Research

\section{Publish your work in this journal}

Cancer Management and Research is an international, peer-reviewed open access journal focusing on cancer research and the optimal use of preventative and integrated treatment interventions to achieve improved outcomes, enhanced survival and quality of life for the cancer patient. The manuscript management system is completely online and includes

\section{Dovepress}

a very quick and fair peer-review system, which is all easy to use. Visit $\mathrm{http}: / /$ www.dovepress.com/testimonials.php to read real quotes from published authors. 